\title{
EDITORIAL (PART II)
}

\section{Assessing the Technology of Developing Health Occupations: Obstacles, Risks, and Strategies for Future Work}

\author{
Nancy T. Watts \\ Massachusetts General Hospital Institute of Health Professions
}

\begin{abstract}
Efforts to expand technology assessment in developing fields, such as physical therapy, face major obstacles. These impediments include scarcity of assessment tools, weak organizational structure for research, and clinicians' reluctance to assess economic factors. Risks that could accompany future work and strategies for reducing problems are discussed.
\end{abstract}

Past efforts to assess health care technology have focused primarily on the work of established professions such as medicine, dentistry, and public health. By contrast, little attention has been given to the technology employed by the many new and rapidly evolving occupations often classified as allied health or paramedical services. Many of these fields have emerged as distinct occupations only within the last several decades, and all are still hard at work defining their roles and attempting to gain recognition from the public and from the established health professions. Charges for the services that these occupations provide have traditionally been quite modest, and their true costs sometimes are obscured by billing practices that incorporate their charges into the basic day rate for hospital care rather than billing for them on a fee-for-service basis. Their newness, diversity, and apparent low cost have blunted concern about the technology of many developing fields on the part of health policy and funding agencies. However, in the 1990s the sheer number of workers in these occupations, their growing involvement in nearly every phase of health care, and the growing total cost of the services that they provide call attention to the need for expanded efforts to assess their technology.

The papers in this special issue illustrate some of the varied approaches such assessments may take. They also highlight some of the problems a field such as physical therapy can expect to encounter in the future. The introduction to this issue described some of the characteristics that many developing fields have in common. A look at the future reveals shared problems as well. Three of these difficulties have been singled out for discussion here because they are serious obstacles to expanding the assessment of the technology of a developing field such as physical therapy. These problems also are of special interest because of the risks that could accompany many efforts to over- 
come them. The developing health care disciplines will need to plot their strategies for the future carefully in order to avoid simply replacing one set of problems with another.

\section{OBSTACLE \#1}

Obstacle \#1 is the scarcity of soundly tested, standardized tools for measuring many important components of care and outcomes of service. While the evaluation of patients has long been an important part of clinical practice in fields such as physical therapy, many of the methods of measurement that are now in use have never been rigorously tested to determine their validity and reliability. Few standardized protocols exist for carrying out even the most widely used test procedures, and little has been done to develop standardized systems for documenting the type and timing of treatment or the characteristics of the individuals who receive care.

These problems are intensified by the difficulty of locating information about testing methods that have been evaluated. This situation is especially obvious if one compares the information on standardized testing procedures available to clinicians and investigators in a field such as physical therapy with that available in a discipline such as psychology. By consulting the latest edition of The Mental Measurements Yearbook (3), an investigator interested in measuring a psychological variable can easily locate descriptions of a large number of different tests, along with information on how each has been evaluated and a critical review of the method's strengths and weaknesses. By contrast, a physical therapist interested in measuring such variables as spasticity, sitting balance, or cough ef fectiveness usually must carry out an independent search of the literature to gather information on any methods of testing that may be available. The recent publication of such references as Rothstein's volume on $\mathrm{Mea}$ surement in Physical Therapy (17) helps greatly, both making critical reviews of existing methods more readily available and stimulating thinking about clinical measurement; but much work remains to be done.

Several of the papers in this special issue provide helpful examples of the careful methodological research needed to develop sound new assessment tools $(5 ; 8 ; 12 ; 15)$. Similar efforts to test and report on the validity and reliability of many other types of measurement will be needed before all of the important aspects of physical therapy technology can be assessed with confidence.

\section{Risks}

As this work proceeds, several other problems could arise, any one of which would seriously limit the value of measurement development projects. They include:

- narrowness of focus: The search for measurement tools that are scientifically respectable could lead to an unbalanced emphasis on variables that are easily quantified and to the neglect of others that are equally important but more difficult to measure. Bioinstrumented recording of isolated physical variables is worthwhile, but many important elements of technology and outcomes of care cannot be measured in this way. One source of this problem was compellingly analyzed some years ago by Feinstein $(6 ; 54-55)$ when he deplored the barriers to clinical science created by such mistaken beliefs as these:

In reasoning, the clinician believes that his intellectual organization of clinical observations is rationally amorphous - that his thinking has too many intricate and unquantified elements to be expressed in the mathematical structures used for other types of scientific analysis. ... In observation, the clinician believes that his descriptions of symptoms and signs cannot be scientifically precise because they often contain nouns, adjectives, verbs, and adverbs rather than numerical dimensions of measurement. 
- duplication of effort: Each of the developing health occupations probably has important concerns that have not attracted the attention of measurement experts in other fields. However, many aspects of health care are of shared interest to a wide variety of disciplines. Much valuable time and effort will be wasted unless efforts to develop new methods of measurement are preceded by an imaginative and thorough review of existing tools. An example of just how useful such borrowed tools can be is presented by Hoskins Michel (9) in her suggestion that a currently undocumented outcome of cardiac rehabilitation could be studied readily using quality-of-life instruments developed for other research.

- limited adoption by clinicians: Even when valuable new measurement methods have been carefully developed, they may remain underused because they are unfamiliar, seem impractical for everyday use in a busy clinic, or call for basic skills in data collection and analysis that few clinicians have. In their report of the disappointing results of a major quality assessment project, Miles-Tapping and Rennie (12) provide a sobering reminder of just how difficult the introduction of a new method of measurement can be.

\section{Strategies}

Future work on measurement methods is most likely to be productive if it is a threepronged effort that incorporates development and testing of new methods and materials, evaluation and improvement of tools already in use in the field, and attempts to locate and adapt methods developed by other disciplines. Within this framework, activities such as the following could help to prevent the problems just described:

- identification of assessment priorities: Planning for new methods of measurement logically begins with consideration of how these measurements are to be used. The first step is to decide what questions a useful assessment of technology should include. These questions then can guide selection of the variables to be measured and the design of the project's measurement tools. Such an approach might reduce the risks of narrowness in methodology and of wasted effort from developing methods that subsequently go unused.

- expanded reporting of measurement projects: Exchange of information both within and among different health care specialties could be greatly facilitated by a variety of clearinghouse mechanisms. State-of-the-art papers and review articles on measurement methodology, multicenter studies and data banks that distribute standardized systems for collecting and reporting data, and compilations of descriptive information on data-gathering instruments all serve this purpose. Even descriptions of instruments that are in rather early stages of development can be useful if they are accompanied by comments on their rationale, the procedure used for development, and apparent strengths and weaknesses. The two-volume compilation of Instruments for Measuring Nursing Practice and Other Health Care Variables (19) published in the late 1970 s to help nurse investigators locate existing research tools is one example of the type of reference material that is needed.

- interdisciplinary projects: Studies such as the one reported in this issue by Anderson et al. (2) allow collaboration by members of both established and developing fields in deciding what assessment questions to address and in designing data-collection instruments. Such studies can help to increase the variety of tools considered and the relevance of the data ultimately collected.

- development of objective methods for measuring subjective phenomena: Such qualitative variables as the nature of interactions between health care providers and their patients, and patients' self-reported symptoms, level of function, and satisfaction with services are too important to ignore. However, they do not lend themselves to measurement using traditional quantitative techniques. Fortunately, alternative methods do exist for measuring such phenomena objectively. References such as Feinstein's Clinimetrics (7) provide guidelines for developing such methods, and several recent papers by physical therapists illustrate their application in that field $(4 ; 11)$. Such measures provide an important complement to such tools as biomedical devices for the collection of data. In combination they allow assessment of 
the full range of variables that are important in health care and also facilitate studies of relationships between "soft" and "hard" data.

- educational resources on measurement: In his review of the development of biofeedback technology, Wolf (21) points out the need for physical therapists to learn more about electronics and computers in order to use these devices wisely. This is only one example of the many topics on which advanced study will be needed if developing technologies are to be wisely used and realistically assessed. Instruction in the basic principles and methods of technology assessment seems especially important, since these have not been emphasized in the basic education of clinicians in many developing fields. Instructional materials and programs ranging from weekend workshops and self-instructional modules to graduate degree programs and postdoctoral fellowships will eventually be needed to meet the growing demand for assessment skills.

\section{Obstacle \#2}

Obstacle \#2 is the lack of a strong organizational structure to support clinical scholarship. The introduction to this issue mentioned the small number of physical therapists who have completed doctoral-level research training (20). The situation is similar in many other developing fields. However, the problem is not one of numbers alone. A constellation of factors inhibits clinical scholarship in these disciplines:

- the environment of research training: In many countries the education of clinicians in fields such as physical therapy has developed largely outside the academic framework of the research university. Even in nations where basic programs are university based, very few postgraduate programs have been established that emphasize theoretical analysis and critical evaluation of the body of knowledge of the clinical discipline itself. As a consequence, many of the members of these occupations who have completed doctoral study have done so in related, but nonclinical, fields such as anatomy, physiology, psychology, or education. Although this undoubtedly has enriched thinking in the clinical discipline, and provided sound training in basic research methodology, this pattern of advanced training often provides little or no opportunity for:

- continued active involvement in direct patient care and development of advanced clinical skills;

- use of research designs and data collection methods selected for their relevance to practical clinical questions;

- guided experience in coping with the many special constraints involved in conducting research with human subjects in highly regulated health care systems;

- identifying and framing research questions directly related to clinical practice in the student's own field;

- receiving the challenge and support of mentors who are knowledgable about both research method and issues of patient care; or

- establishing contact with an active network of seasoned clinical investigators who can continue to stimulate and guide the new scholar during the first years after doctoral study is completed.

- the current job market: In many developing health occupations few jobs offer the new investigator a chance to combine clinical research with patient care responsibilities. Funding for research is limited, demands for productivity in patient service are high, and clinicians with a doctorate often are regarded as overqualified for staff positions. At the same time, the demand for faculty with advanced degrees is strong, especially in those fields whose basic educational programs are based in a university. While faculty in these programs are encouraged to combine research with their teaching, instructional loads usually are heavy, and many programs are located far away from patient care facilities. Many faculty thus find it impossible to continue active practice as clinicians and equally difficult to do research involving patients. 
When they do find time for research it is more likely to emphasize questions, hypotheses, and data collection methods appropriate for studies of normal subjects or laboratory animals than those that are most relevant for clinical studies.

- the limited historical emphasis on research: Although basic instruction in research has been added to the curriculum in many developing fields during recent years, most active clinicians have had little such instruction. Moreover, they often lack any on-the-job contact with active scholars in their own area of practice. Some clinicians in fields such as physical therapy have found opportunities to take part in research by assisting physicians with their studies. However, this participation often consists primarily of carrying out data collection or treatment regimens that are part of a standardized research protocol designed by the physician. Few of these clinicians progress to formulating research questions and designing studies of their own.

- barriers to using reference material: Many clinicians in the developing health occupations practice in settings where access to the research literature is very limited. Most cannot afford to subscribe regularly to more than one or two professional journals; and, except for the relatively small number of staff employed in large health centers and teaching hospitals, most clinicians have little chance to use a health science library. As a consequence, many members of these occupations are unable to keep abreast of research in their field.

\section{Risks}

If the current pattern persists, the outlook for future assessment of technology is troubling. The developing health care occupations could find that the number of individuals with advanced degrees increases substantially, but that their contributions to evaluation and improvement of technology remains very limited. At worst the pattern could become one in which:

- most research is done on healthy, young subjects in a laboratory setting, rather than with patients in a realistic health care environment;

- the clinical skills and concern with the practical questions of direct patient care of most investigators have atrophied from prolonged lack of contact with patients; and

- the results of most studies are published in specialized journals that few clinicians see or understand.

The probability of a widening separation between scholars and clinicians is especially troubling since it carries with it the likelihood that even very relevant research findings may never be translated into improvements in day-to-day patient services.

\section{Strategies}

Fortunately, a variety of powerful antidotes are available to combat these problems. Most have been employed by the established health professions for some time, and many will be very familiar to readers of this journal. Some promising strategies for fostering clinical scholarship include:

- development of advanced degree programs specifically designed to train clinical investigators: The central focus of such programs should be on the technology of the developing health care occupation, with emphasis on examination of clinical theory and on critical assessment of clinical practice. Such programs need not replace the advanced study now done by many clinicians in the basic sciences or in education. However, they would provide a valuable new route for preparation of clinical leaders interested in rationalizing, testing, and improving patient services in their field.

- clinical research centers: Such clusters of investigators actively engaged in research on different facets of a common problem have long been an important feature of the medical research 
scene. Combining research with related patient care and educational programs, these centers of fer rich opportunities for the novice investigator to master clinical research skills under the guidance of a team of seasoned experts. Through linkage with university programs, such centers also of ten make it easier for academic faculty to maintain contact with patients and to focus their research on clinically relevant questions. The participation by investigators from the new health occupations in such centers still is quite limited, but promising examples are beginning to appear. In physical therapy, for example, research groups have been established at locations as diverse as King's College London, McMaster University Medical Centre near Toronto, Washington University in St. Louis, and the medical center at the University of Uppsala in Sweden. Some of these groups are quite informal, others have only piecemeal funding for their work, but all appear to be valuable mechanisms for stimulating clinical research and for encouraging collaboration among scholars from a variety of clinical specialities.

- multicenter studies: Whether their design is epidemiological or experimental, such studies offer several advantages. They allow a large number of active clinicians to gain experience in many phases of research by participating in a study guided by a few experienced investigators. They also facilitate the assessment of clinical technology by helping to overcome the difficulty of securing an appropriately large sample of subjects for descriptive studies and clinical trials. Several of the papers in this issue report on projects that have involved staff from a number of different clinical facilities $(5 ; 8 ; 12 ; 15 ; 18)$. The nature and extent of clinician participation differed widely, but each of these studies clearly benefited from their involvement.

- consensus conferences: Although originally designed primarily to improve utilization of research, such conferences also can provide a valuable forum for identifying research questions and setting research priorities. They may be especially valuable as a catalyst for integrating the complementary skills of experienced clinicians and academic investigators. As with the other mechanisms for fostering clinical research outlined here, the developing health occupations have begun to make use of consensus conferences. For example, in recent years individual physical therapists in Britain, Sweden, the United States, and Canada have been invited to take part in multidisciplinary consensus conferences on topics such as stroke. In addition, many physical and occupational therapists in Sweden have learned how to organize and conduct such conferences on their own by attending intensive workshops in technology assessment in which the consensus conference process is a major topic.

- clinical decision-making tools: These include a wide variety of methods and materials such as clinical algorithms, decision trees, problem-solving flow charts, expert systems, theoretical models, and computer-based guides to patient management. The problem-knowledge couplers described in this issue by Zimny (22) are among the most attractive because of the help that they provide clinicians in using the research literature. In addition to their value as teaching materials, and in guiding the clinicians' work with individual patients, these decision tools all provide important mechanisms for linking technology assessment to practice. They organize and present a broad array of published research in formats that make application to practice easy to visualize. They also help stimulate thinking about which specific research questions most need to be answered. On a more general level, the emphasis that many decision-making systems place on objective definition of the key elements of service and on time-based assessment of outcomes encourages an approach to thinking about technology that makes subsequent attempts at assessment easier.

\section{OBSTACLE \#3}

Obstacle \#3 is the reluctance of many clinicians to assess the economic costs and benefits of their services. In part, this hesitance is probably attributable to lack of information, since clinicians in most health occupations have little instruction in economics during their basic training. However, economic assessment also may be regarded as irrelevant or even distasteful. Concern for the individual patient's wellbeing is the dominant focus of most clinicians' thinking. Studies of costs often are 
seen as the responsibility of bureaucrats and administrators, and fears sometimes arise that they will use cost data as a basis for denying truly needed care to patients. Even in areas of practice where clinicians use economic arguments in marketing their services, discouragingly little has been done to test the reality of these claims. For example, in Australia, Britain, and Scandinavia growing numbers of physical therapists are engaged in programs for the prevention of workplace injuries. Their ergonomic analyses and subsequent recommendations for the redesign of work patterns and equipment are funded by employers in the expectation this will both save money and en hance worker health. However, the long-range impact of such proposed changes and the economic costs and savings associated with them are seldom documented.

Pressure for change in this pattern is mounting. Staff shortages and the rising cost of health care make study of efficiency essential.

\section{Risks}

As clinicians in developing health care occupations give greater attention to the economic side of their services, they will face interesting challenges in deciding how broad a range of factors to assess. Unless these choices are made wisely there is a risk that future cost analyses will be incomplete and misleading. Some of the factors that make cost assessment difficult in the new health disciplines are:

- the prevalence of multidisciplinary care: Many patients who are treated by a specialist such as a physical therapist are concurrently being seen by a variety of other clinicians. Costs and benefits attributable to a single discipline are difficult to tease out. An increased expenditure for service in one area may result in savings in another, but substitutabilities and complementarities among interdependent health disciplines have not been widely studied. Little is known about the effects of different systems for coordinating multidisciplinary services on their effectiveness and cost.

- variations in staff training and experience: Services within any one health care field may be provided by staff with very different levels of expertise. However, few studies have been done to determine what differences in treatment costs and outcomes are seen if services are provided by new graduates rather than experienced staff, clinical specialists rather than generalists, or assistants rather than professional-level clinicians.

- the importance of teaching and long-term care: In many fields, such as dietetics, speech therapy, occupational therapy, and physical therapy, much of the emphasis in treatment is on teaching patients and their families to carry out therapy at home and to comply with recommended changes in lifestyle. Following these recommendations often demands considerable time and effort, and may involve extra expenses for equipment, special food or clothing, or help with homemaking. Many outpatients with chronic disorders receive treatment over a period of months or years. These visits can impose significant expenses of transportation, child care, time away from work, and other activities in order to arrange and gain access to services. However, such related costs are seldom monitored, and determining the economic value of some is difficult.

- the intangible nature of many costs and benefits: Improving the quality of life for patients and their families is often a major goal of treatment. Some improvements are reflected in changes to which it is reasonably easy to attach an economic value (e.g., increased function, earlier return to work), but others are not. This is especially true for services to patients with progressive or severely disabling disorders and to the growing numbers of the elderly. In physical therapy, for example, it is difficult to assign a monetary value to such benefits as reduction of pain for a terminal cancer patient, reduction in the sense of loss and isolation suffered by an alert but elderly stroke patient, or reduction of the frustration and guilt felt by the family of a child with a severe developmental disability. The intangible costs of treatment also may be hard to measure, yet the frustration, embarrassment, or discomfort associated 
with the use of some technologies may make their cost seem prohibitive to some patients. If measurement of costs and benefits is limited to factors that can be valued conveniently in economic terms, an obvious risk emerges that many assessments will be unrealistic from the consumer's point of view.

\section{Strategies}

As in efforts to overcome the first two obstacles, future attempts to increase the attention given to economic aspects of care will call for a multifaceted strategy. Among the activities that seem to be needed are:

- development of guidelines, models, and sample materials: These tools help clinicians and clinical investigators incorporate assessment of economic factors in their planning for individual patients and in research on technology effectiveness. These might range from computer programs that help clinicians compare the probable costs of treatment alternatives for an individual patient, to forms and formulae that could be adapted for use in a variety of cost-effectiveness studies. Projects such as those reported in this issue by Möller, Goldie, and Jonsson (13) will both help to show clinicians the advantages of cost analysis and to provide ideas on how such studies can be designed. Many other examples will be needed to suggest methods for measuring the hidden costs of using services and the intangible rewards and demands that they involve.

- increased educational emphasis on economics: In the future, more instruction on topics such as cost finding, cost control, and cost-effectiveness assessment will be needed as part of the basic curriculum in all health professions. In preparation for this, however, the most immediate need is for workshops, seminars, and independent study materials to help clinicians who are already in practice learn at least the basics of the rationale and methodology of health economics. This program seems most likely to be attractive and useful if it is combined with instruction on methods for assessing and improving the effectiveness of clinical technology. Such classes will be especially valuable if they foster collaboration between health care providers and economists. In the past, clinicians such as physical therapists have learned to work closely with other specialists in fields as diverse as bioengineering, statistics, and pharmacy. It seems more realistic to teach clinicians how to consult productively with an economist than to attempt to make clinical investigators expert economists in their own right.

- health personnel utilization studies: The recent report of a committee appointed by the Institute of Medicine to study the role of allied health personnel in the United States (10) called attention to the need for additional data to answer pressing questions about the most effective and efficient ways to use clinicians in these fields. As Paatero and her colleagues point out (14), the current lack of objective information makes policy making and planning for services such as physical therapy difficult and uncertain. Because many of the developing fields use technology that is highly labor-intensive, studies of the cost-effectiveness of different staffing patterns are especially needed. Because the costs and effectiveness of services provided by one discipline may be strongly influenced by the services provided by others, at least some studies of health personnel will need to be interdisciplinary. Some promising examples of such studies are now in progress to assess the costs and effectiveness of alternative staff mixes for providing home care; however, much additional work of this sort will be needed in the future.

- incentives: Assessing the economic aspects of their work will be a time-consuming task for already busy clinicians, and it is one that may seem complex and unattractive. It will be helpful if the growing demand for such assessment is accompanied by some planned system for allowing clinicians to share in the benefits that this work produces. Some attempts to do this have been made in the past through a variety of profit-sharing programs that pass on to employees part of the revenue generated or expenses saved by their improved productivity. However, these have been linked primarily to increases in the volume of services provided or to its unit cost. A more realistic system would link incentives to treatment effectiveness as well. It also should be recognized that incentives need not be monetary to be appealing. While 
increased earnings would, of course, be popular, many clinicians would also feel rewarded if they could see clear evidence that their efforts at assessment had led to improvements in the services that they provide or to reductions in some of the most frustrating aspects of their own work situation. The unrelenting pace of work and growing demand for services make this an especially important consideration in fields such as physical therapy. The ethical dilemmas inherent in waiting lists, discussed by Purtilo (16), and the concern about their own errors among therapists, studied by Deusinger (5), illustrate a few of the pressures that current practice creates. There should be a strong incentive for clinicians to participate in technology assessment if the improved efficiency that can result from this work allows them greater opportunity to practice in a careful and humanistic way.

\section{CONCLUSION}

Even this brief review of future needs for technology assessment in fields such as physical therapy forewarns us that this work will be complex and challenging. It will call for studies that are inventive and yet build upon the work done by others. While the developing health care occupations have many characteristics that seem to call for new approaches to assessment of their technology, they also have much in common with such established fields as medicine. Both the similarities and differences make the opportunity for physical therapists to contribute to this special issue welcome. The editors hope that in the future many more members of developing health fields will become contributors to this journal as well as avid readers.

\section{AEFERENCES}

1. af Klinteberg, M. Editorial (part I): A summary of the history and present scope of physical therapy. International Journal of Technology Assessment in Health Care, 1992, 8, 4-9.

2. Anderson, J., Campbell, S. K., \& Gardner, H. G. Correlates of physician utilization of physical therapy. International Journal of Technology Assessment in Health Care, 1992, 8, 10-19.

3. Conoley, J. C., \& Kramer, J. J. (eds.). The tenth mental measurements yearbook. Lincoln, NE: The Buros Institute of Mental Measurements, University of Nebraska, 1989.

4. Delitto, A. Subjective measures and clinical decision making. Physical Therapy, 1989, 69, 585-89.

5. Deusinger, S. Analyzing errors in practice: A vehicle for assessing and enhancing the quality of care. International Journal of Technology Assessment in Health Care, 1992, 8, 62-75.

6. Feinstein, A. R. Clinical judgment. Baltimore, MD: Williams \& Wilkins, 1967.

7. Feinstein, A. R. Clinimetrics. New Haven, CT: Yale University Press, 1987.

8. Harrison, M. A., Atkinson, H., \& De Weerdt, W. Benesh movement notation: A tool to record observational assessment. International Journal of Technology Assessment in Health Care, 1992, 8, 44-54.

9. Hoskins Michel, T. Outcome assessment in cardiac rehabilitation. International Journal of Technology Assessment in Health Care, 1992, 8, 76-84.

10. Institute of Medicine Committee to Study the Role of Allied Health Personnel. Allied health services: Avoiding crises. Washington, DC: National Academy Press, 1989.

11. Jette, A. M. Measuring subjective outcomes. Physical Therapy, 1989, 69, 580-84.

12. Miles-Tapping, C., \& Rennie, G. A. The Canadian Physiotherapy Quality of Care Project: Analysis of a derailed project. International Journal of Technology Assessment in Health Care, 1992, 8, 35-43.

13. Möller, G., Goldie, I., \& Jonsson, E. Hospital care versus home care for rehabilitation after hip replacement. Iitternational Journal of Technology Assessment in Health Care, 1992, 8, 93-101.

14. Paatero, H., Holma, T., \& Leisti, S. Crucial questions for physiotherapy in Finland. International Journal of Technology Assessment in Health Care, 1992, 8, 20-25. 
15. Partridge, C. Describing patterns of recovery as a basis for evaluating progress. International Journal of Technology Assessment in Health Care, 1992, 8, 55-61.

16. Purtilo, R. Whom to treat first, and how much is enough? Ethical dilemmas that physical therapists confront as they compare individual patients' needs for treatment. International Journal of Technology Assessment in Health Care, 1992, 8, 26-34.

17. Rothstein, J. M. (ed.). Measurement in physical therapy. New York: Churchill Livingstone, 1985.

18. Torrestad, A., Håkanson, M., \& Axelli, T. The development of a program for the treatment of chronic pain and anxiety: A learning process leading from unsound to sound assessment. International Journal of Technology Assessment in Health Care, 1992, 8, 85-92.

19. Ward, M. J., \& Lindeman, C. A. (eds.). Instruments for measuring nursing practice and other health care variables, vols. 1 and 2. DHEW Publication no. HRA 78-53. Hyatsville, MD: U.S. Department of Health, Education, and Welfare, 1978.

20. Watts, N. T., \& af Klinteberg, M. Introduction. International Journal of Technology Assessment in Health Care, 1992, 8, 1-3.

21. Wolf, S. L. The relationship of technology assessment and utilization: Electromyographic feedback instrumentation as a model. International Journal of Technology Assessment in Health Care, 1992, 8, 102-108.

22. Zimny, N.J. Making information accessible and useful to practicing clinicians: Problemknowledge coupling. International Journal of Technology Assessment in Health Care, 1992, $8,109-117$. 